\title{
Mitochondrial DNA mutations in human tumor cells (Review)
}

\author{
HUI LI ${ }^{1,2}$ and ZE-HUI HONG ${ }^{1,2}$ \\ ${ }^{1}$ Department of Genetics and Developmental Biology, Southeast University School of Medicine; \\ ${ }^{2}$ The Key Laboratory of Developmental Genes and Human Disease, Ministry of Education, \\ Southeast University, Nanjing, Jiangsu 210009, P.R. China
}

Received June 11, 2012; Accepted August 20, 2012

DOI: $10.3892 / \mathrm{ol} .2012 .874$

\begin{abstract}
Mitochondria play significant roles in cellular energy metabolism, free radical generation and apoptosis. The dysfunction of mitochondria is correlated with the origin and progression of tumors; thus, mutations in the mitochondrial genome that affect mitochondrial function may be one of the causal factors of tumorigenesis. Although the role of mitochondrial DNA (mtDNA) mutations in carcinogenesis has been investigated extensively by various approaches, the conclusions remain controversial to date. This review briefly summarizes the recent progress in this field.
\end{abstract}

\section{Contents}

1. Introduction

2. mtDNA mutations in human tumor cells

3. Correlation between mtDNA mutations and tumors

4. Conclusions

\section{Introduction}

Human mitochondrial DNA (mtDNA) is a closed doublestranded circular molecule with a complete sequence of $16,569 \mathrm{bp}$. With the exception of the mature red blood cells, every cell in the body harbors hundreds of mitochondria and thousands of mtDNA molecules. The whole mitochondrial genome encodes 13 essential subunits of the oxidative phosphorylation (OXPHOS) system as well as 2 rRNAs and 22 tRNAs used in the mtDNA translation system $(1,2)$. Strict maternal inheritance, lack of recombination and a high revolutionary rate are genetic characteristics of the mitochondrial genome. Compared with nuclear DNA, mtDNA lacks the

Correspondence to: Dr Hui Li, Department of Genetics and Developmental Biology, Southeast University School of Medicine, Dingjiaqiao Road 87\#, Nanjing, Jiangsu 210009, P.R. China

E-mail: lihui19801019@yahoo.cn; lihui19801019@gmail.com

Key words: mitochondrial DNA, tumor, somatic mutation, germline mutation protective histones and has an extremely inefficient DNA mismatch repair mechanism (3). In addition, a high level of reactive oxygen species (ROS) is generated through the oxidative phosphorylation processes that occur in this organelle and these ROS are prone to damage mtDNA and cause mutations (4). All the above factors confer a high mutation rate: the rate of evolution of the human mitochondrial genome appears to exceed that of the single-copy fraction of the nuclear genome by a factor of approximately 10 (5).

mtDNA mutations that arise in the female germ line are transmitted to the next generation, where they are observed as new mtDNA polymorphisms or as pathogenic mtDNA mutations (6). mtDNA mutations also occur in somatic cells. These somatic mtDNA mutations tend to accumulate with age in postnatal somatic cells, including in brain and skeletal muscle (7). The somatic mtDNA mutations are thought to arise from the oxidative damage caused by the accumulation of ROS in tissues with age and the deficiency of mtDNA repair systems $(3,4)$.

\section{2. mtDNA mutations in human tumor cells}

Tumors pose an increasing threat to human health. Mitochondria play significant roles in cellular energy metabolism and free radical generation and have therefore become a focus of cancer research. Warburg studied the changes of mitochondrial structure and function in tumor cells as early as $1956(8)$. When the role of mitochondria in cellular apoptosis was identified (9), researchers studied the correlation between tumors and the mitochondria and thus began to show interest in the changes of the mitochondrial genome in tumor tissues.

Certain studies have demonstrated that the dysfunction of mitochondria may cause the origin and progression of tumors. For example, certain inherited mutations of nuclear genes that encode proteins which take part in the mitochondrial tricarboxylic acid cycle cause mitochondrial deficiencies and lead to tumor development (10). A fraction of mtDNA mutations identified in human tumor cells are considered to have significant effects on mitochondrial function (11). In summary, the mtDNA mutations causing mitochondrial dysfunction may be the cause of tumorigenesis.

To date, studies on the mutations in the mitochondrial genome have been performed in various tumors. Somatic mtDNA mutations have been reported in various tumor types, 
including breast, colorectal, bladder, gastric, esophageal, lung and oral cancer, head and neck neoplasm and leukemia. These mtDNA mutations include point mutations, deletions and insertions (12-21). The roles that these mtDNA mutations play in tumorigenesis and tumor development have not been determined.

Methods used to detect mtDNA mutations. Somatic mutations are defined as the variants that exist only in the cancerous tissue and not in the corresponding normal tissue. Conversely, the variants that exist in cancerous tissue and in normal tissue are defined as germline mutations. In general, the single-strand conformation polymorphism analysis (SSCP), denaturing high-performance liquid chromatography (DHPLC), temporal temperature gradient electrophoresis (TTGE), as well as direct sequencing (22), which all are based on the PCR method, are used to detect the somatic mtDNA mutations and germline mtDNA mutations. Heteroplasmy occurs when the wild-type and mutant mtDNA coexist in the tissues or cells; otherwise it is named homoplasmy (15).

General features of the mtDNA mutations. The mtDNA mutations identified in various tumor cells or tissues present 3 features that are independent of the type of cancer.

First, the majority of the mtDNA mutations observed are the $\mathrm{T}$ to $\mathrm{C}$ and $\mathrm{G}$ to $\mathrm{A}$ base transitions. This is similar to the mutation pattern of oxidative decay on DNA caused by ROS in normal tissues. Hence, it may be deduced that the main cause of mtDNA mutations in tumors is the elevated high level of ROS (23).

Second, the D-loop region is the mutational hotspot. The D-loop region of human mitochondrial genome is $1,122 \mathrm{bp}$ long. It is the main control area for the duplication and transcription of mtDNA and includes the primary transcript promoter and leading strand for the origin of replication of mtDNA (1). Previous research has revealed the D-loop region as the mutational hotspot in various types of cancer, including breast and bladder cancer and head and neck neoplasms, and also in the normal human population. Therefore, the high mutational frequency in the D-loop region may not lead to the specific changes in tumors. The D-loop is the site that the mtDNA is nearest to the mitochondrial inner membrane and thus is most susceptible to the damage of ROS (24-26).

Third, the majority of the mtDNA mutations detected are homoplasmic. Numerous mtDNA mutations have been reported in various tumor tissues; some were somatic and others were germline mutations (12-21). Of note, the majority of these mutations were homoplasmic. This observation has been interpreted to reflect a replicative advantage for mutated mtDNA copies, or a growth advantage for a cell containing certain mtDNA mutations; thus these mutations may become homoplasmic rapidly during cell fissions and become fixed $(11,15,16)$. However, Coller et al considered that the observed homoplasmy arose entirely by chance in tumor progenitor cells, without any physiological advantage or tumorigenic requirement (28). Through extensive computer modeling, the authors demonstrated that there are sufficient opportunities for a tumor progenitor cell to achieve homoplasmy through unbiased mtDNA replication and sorting during cell division. They revealed that the predicted frequency of homoplasmy in tumor progenitor cells in the absence of selection is similar to the reported frequency of homoplasmic mutations in tumors (27). Additionally, Jones et al reported that the somatic mtDNA mutations in a tumor progenitor cell were capable of undergoing 'genetic drift' to obtain a homoplasmic status through 1,000 or more mitochondrial segregations (28). Chinnery et al also suggested that random genetic drift was sufficiently powerful to explain the homoplasmy and fixation of rare mtDNA mutations in tumor tissues (29).

\section{Correlation between mtDNA mutations and tumors}

Although numerous mtDNA mutations have been reported in various tumor types, it remains unclear what roles they play in tumorigenesis. The question of whether these mtDNA mutations are correlated with tumorigenesis remains controversial. Various researchers hold different opinions. One theory is that the mtDNA mutations simply arise by chance in tumor progenitor cells without any physiological advantage or tumorigenic requirement. By contrast, the other theory states that these mtDNA mutations stimulate tumors and contribute to tumorigenesis and tumor progression.

Theory 1: mtDNA mutations are the byproduct of tumorigenesis. Cancer is a disease induced by multiple factors. Numerous researchers regard the mtDNA mutations in tumors as hitchhike mutations (27-30). They have hypothesized that the mtDNA mutations in malignant cells originated from tumorigenesis. The malignant cells may be in a state of hyperplasia and thus lead to the over-replication of mitochondria. As a result, the mtDNA mutations are generated as a byproduct of tumorigenesis. The following evidence supports this theory:

Firstly, tumor cells have an elevated level of ROS (8). As described above, the majority of the mtDNA mutations in tumor cells are $\mathrm{T}$ to $\mathrm{C}$ and $\mathrm{G}$ to $\mathrm{A}$ base transitions. This is similar to the mutation pattern of oxidative damage to DNA caused by ROS in normal tissues. Hence, it is deduced that the main cause of mtDNA mutations in tumors is the elevated level of ROS (23). The underlying mechanism is similar to a hitchhike mutation: certain factors stimulate the tumorigenesis and at the same time cause an elevated level of ROS. Numerous mtDNA mutations then occur at random as a result of oxidative damage. The D-loop region in tumor cells presents as the mutational hotspot, as is the case in normal tissue, which further demonstrates that these mutations are not specific for tumor cells.

Secondly, over 50 years ago, Warburg pioneered the study of mitochondrial changes in tumor cells and identified that a key event in carcinogenesis involved the development of an injury to the mitochondrial respiratory machinery, resulting in a decrease in oxidative phosphorylation and a compensatory increase in glycolysis that finally led to lactic acidosis (8). It was widely accepted that the tumor cells tended to upregulate glycolysis, in turn leading to less dependence on the mitochondria for oxidative phosphorylation. As a result, the mtDNA mutations did not damage cells in terms of energy metabolism. Certain studies revealed that these mtDNA mutations had the ability to fix or disappear in cells through the mechanism of genetic drift (27-29). These findings further 
support the theory that these mtDNA mutations had no impact on mitochondrial function and thus escaped from the selective pressure of physiological advantage or tumorigenic requirement. Thus, the mtDNA mutations are the byproduct of tumorigenesis.

Finally, Vega et al proposed that the phylogenetic status of mtDNA variation in the context of population genetics was a necessary framework to analyze the correlation between mtDNA variants and carcinogenesis and thus to understand the role that mtDNA variation may play in carcinogenesis (30). In this study, it was suggested that if the mtDNA mutational hotspots detected in tumor cells were common in human populations, the mechanism for their generation should be identical. Therefore, the mtDNA mutations were excluded as the pathogenic factor for tumorigenesis. Following compilation and analysis of the data from previous studies, Vega et al revealed that the majority of the reported tumor-associated mtDNA alterations were common human polymorphisms and mutational hotspots. In conclusion, the authors postulated that the mtDNA mutations in tumor cells were hitchhike mutations and did not play a role in tumorgenesis (30).

Theory 2: mtDNA mutations contribute to tumorigenesis and tumor progression. Certain researchers have suggested that the mtDNA mutations in tumor cells acted as a stimulus to tumors and contributed to tumorigenesis and tumor progression $(12,15,16,31,32)$. The following evidence supports this theory.

Firstly, depletion of mtDNA diminishes the tumorous phenotype. It is well known from the pioneering work of Warburg that tumor cells grown in culture have a higher capacity for glycolysis than non-tumor cells (8). Based on this study, it has been deduced that tumor cells do not completely depend on the mitochondria for oxidative phosphorylation to provide energy. Dani et al stated that the higher glycolytic capacity of tumor cells may be due to the increased growth and nutrient demands of tumor cells, which was not against the theory of the importance of oxidative phosphorylation for the survival and growth of tumors $(33,34)$. It was due to the higher nutrient and oxygen demands that numerous tumors continue to grow in vivo via the induction of neovascularization (35). For example, the study by Knighton et al revealed that during tumor growth there is a vascular and an avascular phase, accompanying various tumor ages and sizes (36). Under the more exacting in vivo circumstances, oxidative phosphorylation coexists with glycolysis and the best adapted cells, which are also the most rapidly proliferating, are those capable of oxidative phosphorylation and glycolysis. In support of this conclusion was the evidence that the depletion of mtDNA diminished the tumorous phenotype (37), indicating that mitochondrial functions were essential for the maintenance of viable tumor cells. The study by Dani et al revealed that the $\Delta$ mtDNA4977 deletion mutation in non-tumoral tissues accumulated with age in somatic cells. However, the apparent absence of this mutation in neoplastic tissues was observed, suggesting that the mitochondrial dysfunction caused by the $\Delta$ mtDNA4977 deletion greatly affected the survival rate of proliferating tumor cells (33). Thus, the mtDNA mutations are not only the hitchhike mutations of tumorigenesis, but the tumor-associated mutations.
Secondly, increased ROS caused by mtDNA mutations contribute to tumorigenesis and tumor progression. A number of tumors have been demonstrated to produce increased ROS (8). Certain studies have implied that the increased ROS production contributes to tumorigenesis and tumor progression. The evidence to support this is that when the superoxide dismutase cDNA was transformed into tumor cells, leading to a decreased ROS production, the tumorous phenotype is reversed (38-40). Other studies revealed that the inhibition of the mitochondrial electron transport chain also increased mitochondrial ROS production (41). Therefore, if mtDNA mutations of the genes encoding subunits of the oxidative phosphorylation system caused mitochondrial dysfunction, they may contribute to tumorigenesis and tumor progression.

Petros et al performed a study in prostate cancer patients that focused on the cytochrome oxidase subunit I (COI) gene and demonstrated that $11-12 \%$ of prostate cancer patients harbored COI mutations that altered conserved amino acids (mean conservation index, 83\%), whereas $<2 \%$ of non-cancerous controls and $7.8 \%$ of the general population harbored COI mutations and the mutations of non-cancerous individuals altered less conserved amino acids (conservation index, 71\%) (42). Therefore, it was proposed that mtDNA mutations that inhibited oxidative phosphorylation increase ROS production and thus contribute to tumorigenicity. To determine whether mutant tumors had increased ROS and tumor growth rates, the authors introduced the pathogenic mtDNA ATP6 T8993G mutation into the PC3 prostate cancer cell line through cybrid transfer and tested the tumor growth in nude mice. The mutant (T8993G) cybrids were demonstrated to generate tumors that were 7 times larger than the wild-type (T8993T) cybrids, whereas the wild-type cybrids barely grew in the mice. The mutant tumors also generated significantly more ROS, while the wild-type had no changes. Therefore, Petros et al suggested that mtDNA mutations generated elevated ROS levels that had a positive correlation with the tumor growth rate. In summary, this study supported the conclusion that mtDNA mutations contribute to tumorigenesis and tumor progression by producing high amounts of ROS (42).

Finally, mtDNA mutations promote tumors by preventing apoptosis. Shidara et al revealed that pathogenic mutations in the mitochondrial genome appeared to promote tumorigenesis and tumor progression. The authors suggested that these mtDNA mutations contributed to tumorigenesis and tumor progression by preventing apoptosis (43). In their study, Shidara et al constructed cybrids with a homoplasmic pathogenic point mutation in the mitochondrial genome and constructed cybrids without the above mtDNA mutations against an identical nuclear background. When the cybrids were transplanted into nude mice, the mutant conferred an advantage in the promotion of cancer and in the early stage of tumor growth.

To study the faster growth of tumors derived from the mutant cybrids, the authors compared mutant and wild-type cybrids in terms of their increasing rates in culture. The mutant cybrids increased faster than wild-type in culture. To explore the molecular mechanism underlying the observations, they detected the cell apoptosis frequency in cybrids. The data revealed that mutant cybrids had a lower frequency of 
apoptosis than wild-type cybrids (43). Therefore, the authors suggested that the mtDNA mutations hindered cell apoptosis which promoted tumorigenesis and tumor progression.

\section{Conclusions}

The role of mtDNA mutations in carcinogenesis has been investigated extensively by various approaches and remains controversial. Constructing the cell cybrids would be the most efficient method to clarify the correlation between mtDNA mutations and tumorigenesis. By constructing the cybrids containing mutant mtDNA cells and $\varrho 0$ cells devoid of mtDNA or transforming the mutant mtDNA into $\mathrm{Q} 0$ cells, a series of related analyses may be performed, including growth rates, cell behaviors and the functions in mitochondria replication. However, previously reported cybridization experiments have a common drawback in that they did not use genuine mutant mtDNAs derived from actual cancer tissues $(42,43)$. As a result, the conclusions obtained are inevitably limited.

The mtDNA mutations exert their pathogenicity through the changes in RNAs and proteins they encoded. The mitochondria proteomic analysis would be critical in an mtDNA-related etiology study (44). Although the mitochondrial function proteomic analysis is still in its infancy, it is likely to aid the elucidation of the role that mitochondria play in tumorigenesis as well as identifying potential targets for therapeutic intervention in the future.

\section{Acknowledgements}

We would like to acknowledge Dr Liang Xie and Dr Hui Pan for their assistance in drafting the manuscript. This work was supported by grants from Science \& Technology of Southeast University (KJ2010439) and the National Natural Science Foundation of China (31000955).

\section{References}

1. Anderson S, Bankier AT, Barrell BG, et al: Sequence and organization of the human mitochondrial genome. Nature 290 457-465, 1981.

2. Andrews RM, Kubacka I, Chinnery PF, Lightowlers RN, Turnbull DM and Howell N: Reanalysis and revision of the Cambridge reference sequence for human mitochondrial DNA. Nat Genet 23: 147, 1999.

3. Croteau DL and Bohr VA: Repair of oxidative damage to nuclear and mitochondrial DNA in mammalian cells. J Biol Chem 272: 25409-25412, 1997.

4. Yakes FM and Van Houten B: Mitochondrial DNA damage is more extensive and persists longer than nuclear DNA damage in human cells following oxidative stress. Proc Natl Acad Sci USA 94: 514-519, 1997.

5. Brown WM, George M Jr and Wilson AC: Rapid evolution of animal mitochondrial DNA. Proc Natl Acad Sci USA 76: 1967-1971, 1979.

6. Cavelier L, Jazin E, Jalonen P and Gyllensten U: MtDNA substitution rate and segregation of heteroplasmy in coding and noncoding regions. Hum Genet 107: 45-50, 2000.

7. Wallace DC: Mitochondrial DNA sequence variation in human evolution and disease. Proc Natl Acad Sci USA 91: 8739-8746, 1994.

8. Warburg O: On the origin of cancer cells. Science 123: 309-314, 1956.

9. Green DR and Reed JC: Mitochondria and apoptosis. Science 281: 1309-1312, 1998.
10. Eng C, Kiuru M, Fernandez MJ and Aaltonen LA: A role for mitochondrial enzymes in inherited neoplasia and beyond. Nat Rev Cancer 3: 193-202, 2003.

11. Penta JS, Johnson FM, Wachsman JT and Copeland WC: Mitochondrial DNA in human malignancy. Mutat Res 488: 119-133, 2001.

12. Habano W, Sugai T, Yoshida T and Nakamura S: Mitochondrial gene mutation, but not large-scale deletion, is a feature of colorectal carcinomas with mitochondrial microsatellite instability. Int J Cancer 83: 625-629, 1999.

13. Abnet CC, Huppi K, Carrera A, et al: Control region mutations and the 'common deletion' are frequent in the mitochondrial DNA of patients with esophageal squamous cell carcinoma. BMC Cancer 4: 30, 2004.

14. Aikhionbare FO, Khan M, Carey D, Okoli J and Go R: Is cumulative frequency of mitochondrial DNA variants a biomarker for colorectal tumor progression? Mol Cancer 3: 30, 2004.

15. Polyak K, Li Y, Zhu H, et al: Somatic mutations of the mitochondrial genome in human colorectal tumours. Nat Genet 20: 291-293, 1998.

16. Fliss MS, Usadel H, Caballero OL, et al: Facile detection of mitochondrial DNA mutations in tumors and bodily fluids. Science 287: 2017-2019, 2000.

17. Hibi K, Nakayama H, Yamazaki T, et al: Detection of mitochondrial DNA alterations in primary tumors and corresponding serum of colorectal cancer patients. Int J Cancer 94: 429-431, 2001.

18. Linnartz B, Anglmayer R and Zanssen S: Comprehensive scanning of somatic mitochondrial DNA alterations in acute leukemia developing from myelodysplastic syndromes. Cancer Res 64: 1966-1971, 2004.

19. Kumimoto H, Yamane Y, Nishimoto Y, et al: Frequent somatic mutations of mitochondrial DNA in esophageal squamous cell carcinoma. Int J Cancer 108: 228-231, 2004.

20. Wang CY, Li H, Hao XD, et al: Uncovering the profile of somatic mtDNA mutations in Chinese colorectal cancer patients. PLoS One 6: e21613, 2011.

21. Wang CY, Wang HW, Yao YG, Kong QP and Zhang YP: Somatic mutations of mitochondrial genome in early stage breast cancer. Int J Cancer 121: 1253-1256, 2007.

22. Bi R, Li WL, Chen MQ, Zhu Z and Yao YG: Rapid identification of mtDNA somatic mutations in gastric cancer tissues based on the mtDNA phylogeny. Mutat Res 709-710: 15-20, 2011.

23. Beckman KB and Ames BN: Oxidative decay of DNA. J Biol Chem 272: 19633-19636, 1997.

24. Vanecek T, Vorel F and Sip M: Mitochondrial DNA D-loop hypervariable regions: Czech population data. Int J Legal Med 118: 14-18, 2004.

25. Lutz S, Weisser HJ, Heizmann J and Pollak S: Location and frequency of polymorphic positions in the mtDNA control region of individuals from Germany. Int J Legal Med 111: 67-77, 1998.

26. Stoneking M: Hypervariable sites in the mtDNA control region are mutational hotspots. Am J Hum Genet 67: 1029-1032, 2000.

27. Coller HA, Khrapko K, Bodyak ND, Nekhaeva E, Herrero-Jimenez P and Thilly WG: High frequency of homoplasmic mitochondrial DNA mutations in human tumors can be explained without selection. Nat Genet 28: 147-150, 2001.

28. Jones JB, Song JJ, Hempen PM, Parmigiani G, Hruban RH and Kern SE: Detection of mitochondrial DNA mutations in pancreatic cancer offers a 'mass'-ive advantage over detection of nuclear DNA mutations. Cancer Res 61: 1299-1304, 2001.

29. Chinnery PF, Samuels DC, Elson J and Turnbull DM: Accumulation of mitochondrial DNA mutations in ageing, cancer, and mitochondrial disease: is there a common mechanism? Lancet 360: 1323-1325, 2002.

30. Vega A, Salas A, Gamborino E, Sobrido MJ, Macaulay V and Carracedo A: mtDNA mutations in tumors of the central nervous system reflect the neutral evolution of mtDNA in populations. Oncogene 23: 1314-1320, 2004.

31. Kloss-Brandstätter A, Schäfer G, Erhart G, et al: Somatic mutations throughout the entire mitochondrial genome are associated with elevated PSA levels in prostate cancer patients. Am J Hum Genet 87: 802-812, 2011.

32. Tseng LM, Yin PH, Yang CW, et al: Somatic mutations of the mitochondrial genome in human breast cancers. Genes Chromosomes Cancer 50: 800-811, 2011.

33. Dani MA, Dani SU, Lima SP, et al: Less deltamtDNA4977 than normal in various types of tumors suggests that cancer cells are essentially free of this mutation. Genet Mol Res 3: 395-409, 2004. 
34. Bai RK, Chang J, Yeh KT, et al: Mitochondrial DNA content varies with pathological characteristics of breast cancer. J Oncol 2011: 496189, 2011.

35. Brooks PC, Montgomery AM, Rosenfeld M, et al: Integrin alpha $v$ beta 3 antagonists promote tumor regression by inducing apoptosis of angiogenic blood vessels. Cell 79: 1157-1164, 1994.

36. Knighton D, Ausprunk D, Tapper D and Folkman J: Avascular and vascular phases of tumour growth in the chick embryo. Br J Cancer 35: 347-356, 1977.

37. Hayashi J, Takemitsu M and Nonaka I: Recovery of the missing tumorigenicity in mitochondrial DNA-less HeLa cells by introduction of mitochondrial DNA from normal human cells. Somat Cell Mol Genet 18: 123-129, 1992.

38. McCord JM: The evolution of free radicals and oxidative stress. Am J Med 108: 652-659, 2000.

39. Baker AM, Oberley LW and Cohen MB: Expression of antioxidant enzymes in human prostatic adenocarcinoma. Prostate 32: 229-233, 1997.
40. Bostwick DG, Alexander EE, Singh R, et al: Antioxidant enzyme expression and reactive oxygen species damage in prostatic intraepithelial neoplasia and cancer. Cancer 89: 123-134, 2000.

41. Esposito LA, Melov S, Panov A, Cottrell BA and Wallace DC: Mitochondrial disease in mouse results in increased oxidative stress. Proc Natl Acad Sci USA 96: 4820-4825, 1999.

42. Petros JA, Baumann AK, Ruiz-Pesini E, et al: mtDNA mutations increase tumorigenicity in prostate cancer. Proc Natl Acad Sci USA 102: 719-724, 2005.

43. Shidara Y, Yamagata K, Kanamori T, et al: Positive contribution of pathogenic mutations in the mitochondrial genome to the promotion of cancer by prevention from apoptosis. Cancer Res 65: 1655-1663, 2005

44. Verma M, Kagan J, Sidransky D and Srivastava S: Proteomic analysis of cancer-cell mitochondria. Nat Rev Cancer 3: 789-795, 2003. 